\title{
CA19-9 as a therapeutic target in pancreatitis
}

\author{
Shingo Kato ${ }^{1}$, Kazufumi Honda ${ }^{2}$ \\ ${ }^{1}$ Department of Clinical Cancer Genomics, Yokohama City University, Yokohama, Japan; ${ }^{2}$ Department of Biomarkers for Early Detection of Cancer, \\ National Cancer Center Research Institute, Tokyo, Japan \\ Correspondence to: Shingo Kato, MD, PhD. A467, 3-9, Fukuura, Kanazawa-ku, Yokohama 236-0004, Japan. Email: shin800m@yokohama-cu.ac.jp. \\ Provenance: This is an invited article commissioned by the Section Editor Dr. Le Li (Department of Pancreatic and Biliary Surgery, The First \\ Affiliated Hospital of Harbin Medical University, Harbin Medical University, Harbin, China). \\ Comment on: Engle DD, Tiriac H, Rivera KD, et al. The glycan CA19-9 promotes pancreatitis and pancreatic cancer in mice. Science 2019;364:1156-62.
}

Submitted Sep 15, 2019. Accepted for publication Sep 29, 2019.

doi: 10.21037/atm.2019.09.161

View this article at: http://dx.doi.org/10.21037/atm.2019.09.161

The glycan carbohydrate antigen 19-9 (CA19-9) is one of the most important and widely used biomarkers for pancreatic cancer. In 1979, Koprowski et al. first described CA 19-9 as a tumor antigen recognized by the monoclonal antibody NS19-9 (1). NS19-9 was developed by hybridoma technology using the human colorectal cancer cell line SW1116 as an immunizing antigen. The antigen recognized by NS19-9 is a type of Lewis blood group on the cell membrane surface, and is a sialyl Lewis A antigen $\left(\mathrm{sLe}^{\mathrm{a}}\right)$ in which a sialic acid is added to the Lewis A sugar chain $\left(\mathrm{Le}^{\mathrm{a}}\right)$. During the generation of sLe ${ }^{\mathrm{a}}$, an enzyme called 1,4-fucosyl transferase is required for adding fucose to its precursor, sialyl $\mathrm{Le}^{\mathrm{c}}$. One of the most important limitations of CA199 as a tumor marker is that $5-10 \%$ of population lacks this enzyme and as a result cannot produce CA19-9.

Although CA19-9 is useful for monitoring treatment response and as a marker of recurrent disease, this antigen is considered not to be appropriate for mass screening of asymptomatic patients (2). Satake et al. assessed serum CA 19-9 levels in 12,840 asymptomatic individuals and 8,706 symptomatic outpatients in Japan. As a result, among 12,840 asymptomatic individuals, only four pancreatic cancer cases were identified (3). In contrast, 104 patients were diagnosed with pancreatic cancer among 8,706 symptomatic patients. In another study, Kim et al. examined serum CA 19-9 levels in 70,940 asymptomatic individuals in Korea, and identified only 4 patients with pancreatic cancer (4). Because of such low detection rates of pancreatic cancer and the fact that elevated serum levels of CA19-9 also can be found in several other types of cancers and in a number of benign diseases including pancreatitis (5), CA19-9 is not commonly used for a mass screening for pancreatic cancer.

The biological function of CA19-9 is largely unknown. It has been reported that E-selectin expressed by endothelial cells is an endogenous receptor of CA19-9 and may play a role in cancer invasion/metastasis $(6,7)$. However, since Fucosyltransferase 3 (FUT3), which encodes 1,4-fucosyl transferase, is a pseudogene in rodents, it has been difficult to analyze the role of CA19-9 in murine disease models (8). To assess the biological function of CA19-9 in the pancreas, Engle et al. created transgenic mice that expressed CA19-9. Using this novel experimental tool, they recently reported that production of CA19-9 in mice promotes pancreatitis and cancer in mice (9). This study revealed the utility of CA19-9, which has long been used as a tumor marker, as a potential therapeutic target.

The study included the careful examination of several basic issues. First, since CA19-9 is a glycan that does not normally exist in mice, it was unclear whether CA19-9 is expressed in a normal conformation after the introduction of the human genes related to its synthesis. Accordingly, introduction of FUT3 alone was insufficient for murine pancreatic cancer cells to produce CA19-9. Instead of CA19-9, the expression of Lewis ${ }^{\mathrm{x}}$ antigen was increased after FUT3 introduction. Considering that Lewis ${ }^{\mathrm{x}}$ antigen is generated from type II chain precursors and that CA199 is produced from type I chain precursors, reprogramming of the precursor substrates would be necessary for murine pancreatic cancer cells to produce CA19-9. Thus, a gene encoding 1,3-galactosyltransferase 5 (3GALT5), which is required for the production of type I chain precursors, was introduced in combination with FUT3. Transfection 
of mouse pancreatic cancer cells with the genes encoding both FUT3 and B3GALT5 successfully led to cell surface production of CA19-9. Additionally, CA19-9 protein carriers in the mouse cells harboring the human FUT3 and B3GALT5 genes were identified by mass spectrometry. This analysis demonstrated that expression of the human FUT3 and B3GALT5 genes in mouse cells largely recapitulates the human CA19-9 carrier profile. These initial validations of in vitro analysis were important to ensure that the introduction of CA19-9 expression in murine tissues mimics the antigen's behavior in human tissues.

Another basic concern was that when CA19-9 production is induced in mouse tissue, this exogenous glycan may cause an excessive autoimmune response in multiple tissues. To address this question, the authors generated two transgenic mouse lines with inducible CA19-9 production. They created transgenic mice that expressed CA19-9 throughout the body (CA19-9/whole) and mice that expressed CA199 only in the pancreas, duodenum, and bile ducts (CA199/PDX1-cre). These two transgenic mouse lines facilitated better understanding of the biological behavior of CA199 in vivo. Surprisingly, although both lines demonstrated histologic signs of pancreatitis, all other tissues except the pancreas appeared histologically normal after induction of gene expression [by doxycycline (Dox) treatment]. These findings suggested that although CA19-9 is used as a tumor marker in several cancer types, CA19-9 is pathogenic only in the pancreas.

These transgenic mouse models mimicked the clinical characteristics of human acute and chronic pancreatitis. The histology of the pancreas following CA19-9 production demonstrated interstitial edema, lymphocyte infiltration, and collagen deposition. Both mouse lines demonstrated elevated serum levels of the pancreatic enzymes amylase and lipase, and the levels of these enzymes normalized in the chronic phase (after 4 weeks of Dox treatment). These changes of serum levels of pancreatic enzymes over time are similar to those observed in human acute and chronic pancreatitis. In addition to the effects on the serum levels of pancreatic enzymes, the CA19-9 expression pattern in the pancreas was similar between the two CA19-9 mouse lines and human patients. In both mouse lines, CA19-9 was expressed predominantly in pancreatic ducts and islet cells, and not in acinar cells.

The authors also revealed the molecular mechanisms of CA19-9-induced pancreatitis using pancreatic ductal organoids grown from CA19-9/PDX1-cre mice. They focused on epidermal growth factor receptor (EGFR) signaling since EGFR has been shown to be necessary and sufficient for the induction of pancreatitis $(10,11)$. After induction of CA19-9 expression, elevation of EGFR phosphorylation (at Y1068 and Y1148) was observed in CA19-9/PDX1-cre organoids. Interestingly, this CA19-9-mediated increase in phosphorylated EGFR was not induced by the receptor's general ligand, EGF. Although this increase in phosphorylated EGFR induced by CA19-9 was accompanied by increased phosphorylation of focal adhesion kinase (FAK) (at Y397), EGFR stimulation by EGF did not induce FAK phosphorylation. On the other hand, conditioned medium from CA19-9-expressing organoids stimulated EGFR phosphorylation in control murine ductal organoids. These finding suggested the existence of a soluble ligand that mediates EGFR stimulation. To identify this unknown ligand, EGFR complexes from CA19-9/ PDX1-cre organoid lysates were immunoprecipitated (IPed) and identified by mass spectrometry (MS). This analysis revealed fibulin-3 (FBLN3) as the soluble ligand, and the authors demonstrated that FBLN3 is necessary for the activation of the EGFR pathway after CA19-9 expression. Multiple FBLN3-blocking antibodies prevented the phosphorylation of EGFR and the activation of downstream effector pathways in CA19-9/PDX1-cre organoids.

The mechanism of CA19-9-mediated pancreatitis in this model is very interesting. Since CA19-9 is an exogenous antigen in mice, immune system-mediated pancreatitis is hypothesized to be the primary mechanism in this model. When CA19-9 expression is induced by Dox treatment, the antigen must immediately be detected by innate immunity. The authors showed that, as expected, the infiltration of macrophages into the pancreas increased after induction of CA19-9 expression in this model. While the innate immune system responds to the CA19-9 expressing cells, anti-CA19-9 antibody also must be created. The resulting effector cells then are expected to attack CA19-9-expressing cells by antibody-dependent cell-mediated cytotoxicity. In human pancreatitis, it has been reported that macrophages are the predominant class of cells that infiltrate the pancreas during the acute phase of the disease; after progression to a chronic condition, $\mathrm{T}$ and $\mathrm{B}$ cells also infiltrate the pancreas (12). In this CA19-9-mediated murine pancreatitis model, $\mathrm{T}$ and $\mathrm{B}$ cell infiltration also was observed in the chronic phase of pancreatitis. However, in this model, pancreatitis was induced by a soluble factor, FBLN3, which is secreted by CA19-9 expressing pancreatic cells. Given that FBLN3-blocking antibodies prevented EGFR phosphorylation in CA19-9/PDX1-cre organoids, FBLN3 
is a possible therapeutic target. It will be interesting to assess whether FBLN3-blocking antibodies can counteract CA19-9 mediated pancreatitis in this model. Also, determination of the possible role of FBLN3 in human pancreatitis will be needed.

One of the most intriguing parts of this study is that the authors showed the possibility of CA19-9 as a therapeutic target in pancreatitis. Since CA19-9-induced pancreatitis was attenuated after stopping Dox administration, it appears that CA19-9 expression is required to maintain pancreatitis in this model. Interestingly, treatment with anti-CA19-9 antibody reduced serum amylase levels and normalized the pancreatic histology. These data suggested that CA19-9 plays a role in disease pathogenesis and maintenance and that CA19-9-targeted therapy may warrant further exploration. The authors also examined inhibition of EGFR in vivo by using erlotinib in this model. However, erlotinib was not as effective as CA19-9 antibody blockade in mitigating pancreatitis induction in this mouse model. As mentioned before, anti-CA19-9 antibody must be generated after the induction of CA19-9 expression. The therapeutic effect of anti-CA19-9 antibody administration suggests that induced CA19-9s are not saturated by the newly created anti-CA19-9 antibody in the host mouse.

Finally, the authors examined the effect of CA19-9mediated pancreatitis on pancreatic tumorigenesis. They created transgenic mice that express oncogenic Kras (G12D) and CA19-9 in the pancreas (Kras/CA19-9/PDX1cre mice). It has been reported that transgenic mice that express oncogenic Kras (G12D) in the pancreas (Kras/ PDX1-cre mice) develop pre-cancerous lesions (pancreatic intraepithelial neoplasias; PanINs) and invasive cancer. In the Kras/PDX1-cre model, both the total number of PanINs and their grade increased with the advancing age of the mice, and it took approximately 6 months to develop invasive cancer (13). Dox treatment of Kras/ CA19-9/PDX1-cre mice demonstrated that the additional CA19-9 expression significantly accelerated pancreatic tumorigenesis. Importantly, widespread metastases were observed in the peritoneum, diaphragm, liver, and lung in multiple Dox-treated Kras/CA19-9/PDX1-cre mice. These findings suggested that CA19-9 induces pancreatitis or that CA19-9 itself accelerates pancreatic tumorigenesis and make cancer cells more aggressive. This increased aggressiveness is very interesting. It has been reported that it takes several months for Kras/PDX1-cre mice to develop invasive cancers, and that additional mutations in tumor-suppressor genes such as TP53 (14) or CAKN2A (15) accelerates pancreatic tumorigenesis and increases aggressiveness. In the Kras/CA19-9/PDX1-cre mouse model, highly aggressive pancreatic cancer with multiple organ metastases was induced without additional introduction of mutations in tumor-suppressor genes. It will be very interesting to see what genetic alterations occur in this cancer.

This study demonstrated the possibility of CA19-9 as a therapeutic target for the treatment of pancreatitis and pancreatic cancer. At present, there is no diagnostic method for identifying the cause of pancreatitis, with the exception of gallstone pancreatitis. On the contrary, there are still many types of pancreatitis with unknown etiology. This study is important not only for our better understanding of the underlying mechanism of pancreatitis but also for clinical applications. As the authors mention in the manuscript, Immuno-Positron Emission Tomography with fully humanized anti-CA19-9 antibodies have passed Phase 1A clinical trials for pancreatic cancer (NCT02687230). If such antibodies become available for clinical use, the expression level of tissue CA19-9 in living humans could be assessed, providing a molecular imaging modality that may facilitate the development of improved strategies for the treatment of pancreatitis.

\section{Acknowledgments}

None.

\section{Footnote}

Conflicts of Interest: The authors have no conflicts of interest to declare.

Ethical Statement: The authors are accountable for all aspects of the work in ensuring that questions related to the accuracy or integrity of any part of the work are appropriately investigated and resolved.

\section{References}

1. Koprowski H, Steplewski Z, Mitchell K, et al. Colorectal carcinoma antigens detected by hybridoma antibodies. Somatic Cell Genet 1979;5:957-71.

2. Fahrmann JF, Bantis LE, Capello M, et al. A PlasmaDerived Protein-Metabolite Multiplexed Panel for Early-Stage Pancreatic Cancer. J Natl Cancer Inst 2019;111:372-9.

3. Satake K, Takeuchi T, Homma T, et al. CA19-9 as a 
screening and diagnostic tool in symptomatic patients: the Japanese experience. Pancreas 1994;9:703-6.

4. Kim JE, Lee KT, Lee JK, et al. Clinical usefulness of carbohydrate antigen 19-9 as a screening test for pancreatic cancer in an asymptomatic population. J Gastroenterol Hepatol 2004;19:182-6.

5. Ventrucci M, Pozzato P, Cipolla A, et al. Persistent elevation of serum CA 19-9 with no evidence of malignant disease. Dig Liver Dis 2009;41:357-63.

6. Takada A, Ohmori K, Yoneda T, et al. Contribution of carbohydrate antigens sialyl Lewis A and sialyl Lewis $\mathrm{X}$ to adhesion of human cancer cells to vascular endothelium. Cancer Res 1993;53:354-61.

7. Kannagi R. Carbohydrate antigen sialyl Lewis a-its pathophysiological significance and induction mechanism in cancer progression. Chang Gung Med J 2007;30:189-209.

8. Xia B, Feasley CL, Sachdev GP, et al. Glycan reductive isotope labeling for quantitative glycomics. Anal Biochem 2009;387:162-70.

9. Engle DD, Tiriac H, Rivera KD, et al. The glycan CA199 promotes pancreatitis and pancreatic cancer in mice.

Cite this article as: Kato S, Honda K. CA19-9 as a therapeutic target in pancreatitis. Ann Transl Med 2019;7(Suppl 8):S318. doi: 10.21037/atm.2019.09.161
Science 2019;364:1156-62.

10. Ardito CM, Gruner BM, Takeuchi KK, et al. EGF receptor is required for KRAS-induced pancreatic tumorigenesis. Cancer Cell 2012;22:304-17.

11. Navas C, Hernandez-Porras I, Schuhmacher AJ, et al. EGF receptor signaling is essential for k-ras oncogenedriven pancreatic ductal adenocarcinoma. Cancer Cell 2012;22:318-30.

12. Habtezion A. Inflammation in acute and chronic pancreatitis. Curr Opin Gastroenterol 2015;31:395-9.

13. Hingorani SR, Petricoin EF, Maitra A, et al. Preinvasive and invasive ductal pancreatic cancer and its early detection in the mouse. Cancer Cell 2003;4:437-50.

14. Hingorani SR, Wang L, Multani AS, et al. Trp53R172H and KrasG12D cooperate to promote chromosomal instability and widely metastatic pancreatic ductal adenocarcinoma in mice. Cancer Cell 2005;7:469-83.

15. Aguirre AJ, Bardeesy N, Sinha M, et al. Activated Kras and Ink4a/Arf deficiency cooperate to produce metastatic pancreatic ductal adenocarcinoma. Genes Dev 2003;17:3112-26. 